Produto \& Produção, vol. 14 n.1, p.22-39, fev. 2013

RECEBIDO EM 13/07/2012. ACEITO EM 06/09/2012.

\title{
Aplicação da simulação computacional para determinar a capacidade produtiva do processo de produção de pães: um estudo de caso
}

\author{
Isaac Pergher \\ Centro Universitário Univates, Lajeado, $R S$ \\ Faculdades FTEC, Caxias do Sul, RS \\ eng.isaac@hotmail.com
}

\section{Guilherme Luís Vaccaro}

Universidade do Vale do Rio dos Sinos - UNISINOS, São Leopoldo, RS

guilhermev@unisinos.br

\author{
Maricelse Pradella \\ Universidade do Vale do Rio dos Sinos - UNISINOS, São Leopoldo, RS \\ maricelse@bol.com.br
}

\section{RESUMO}

Nos últimos anos, devido aos requisitos que os sistemas de produção devem atender, ao projetar ou dimensionar um sistema produtivo, tem aumentado a importância de estimar, a priori, o comportamento de gargalos, filas, índices de produtividade, entre outros. Diante disto, a simulação computacional tem-se mostrado bastante eficaz como ferramenta de auxílio no projeto de processos industriais. Neste trabalho, apresenta-se uma aplicação da simulação computacional, com o objetivo de determinar a capacidade produtiva de um processo de produção de pães, projetada para atender as exigências de um nicho de mercado consumidor. No modelo de simulação foram analisados três cenários denominados $\mathrm{A}, \mathrm{B}$ e C que foram configurados a partir das recomendações dos gestores do processo e pelos autores deste estudo. O método de condução de projetos utilizado é baseado no preconizado por Law e Kelton (2000). Os principais resultados obtidos referem-se à adequação da capacidade dos equipamentos para atendimento da demanda, respeitando requisitos técnicos de perecibilidade dos produtos, prazos de entrega e requisitos específicos de qualidade.

Palavras-chave: simulação computacional; capacidade produtiva; indústria alimentícia.

\begin{abstract}
In recent years has increased the importance of estimating a priori the behavior of bottlenecks, queues, productivity rates, among others when designing a production system. Given this the computer simulation has proved quite effective as a tool to aid in the design of industrial processes. This paper presents an application of computer simulation in order to determine the productive capacity of a production process of breads designed to meet the demands of a niche consumer market. In the simulation model were analyzed three scenarios named A, B and $\mathrm{C}$ that have been configured from the recommendations of the management process and the authors of this study. The driving method used is based on designs recommended by Law and Kelton (2000). The main results concern the adequacy of equipment capacity to meet
\end{abstract}


demand, respecting technical requirements of perishable products, delivery and specific quality requirements.

Keywords: computer simulation; production capacity; food industry.

\section{Introdução}

O aumento da complexidade dos mercados, fruto da globalização e da alteração das relações de governança das cadeias de produção e consumo tem exigido continuamente das organizações, o desenvolvimento de estratégias voltadas à obtenção de vantagem competitiva, no intuito obter uma posição de destaque de sua concorrência e aumentar sua participação no mercado ou garantir sustentabilidade.

Conforme Klippel et al. (2007), no Brasil, as condições competitivas foram enfrentadas, basicamente, através da adoção de princípios de Engenharia de Produção, tais como: Sistema Toyota de Produção, Qualidade Total, Teoria das Restrições, entre outros, visando à busca pela racionalização dos recursos. Contudo, é possível observar na literatura que o desenvolvimento dessas metodologias foi subsidiado por organizações que produziam bens duráveis, ou mais especificamente, não eram enquadras no segmento alimentício. A temática da competitividade e a busca pela racionalização dos recursos não é diferente na indústria de alimentos, tornando-se mais importante, na medida em que se considera a perecibilidade dos produtos, como é o caso dos panifícios.

Frente à necessidade de abastecer um mercado consumidor, formado por redes de supermercados, refeitórios de empresas e licitações de prefeituras (para o fornecimento de pães em escolas, creches e orfanatos), as empresas panificadoras necessitam estar aptas para atender demandas de diferentes quantidades e modelos de produtos, respeitando prazos de entrega e padrões sanitários e de qualidade. Esses padrões traduzem-se tecnicamente em níveis de atendimento compatíveis com as características físico-químicas dos produtos. Um elemento de destaque é a variabilidade sobre a qual os processos podem estar sujeitos, podendo causar impacto direto sobre a perecibilidade e a degradação do produto in natura. Este contexto demanda um adequado dimensionamento da capacidade produtiva em relação aos volumes de massa a serem processados. Diante dessa realidade, uma empresa que deseja atuar nesse nicho de mercado deve ser capaz de gerir sua capacidade de produção, para concentrar ações visando a adequar o processo produtivo para cumprir os prazos de entrega e oferecer flexibilidade no atendimento das solicitações inesperadas.

Neste contexto, o presente trabalho descreve um estudo de caso que trata da determinação da capacidade produtiva de um panifício, tendo enfoque no atendimento das exigências de um mercado consumidor previamente estabelecido e com potencial de expansão. Esta capacidade foi dimensionada com base em uma abordagem de simulação computacional, fundamentada no método preconizado por Law e Kelton (2000). A pesquisa realizada utilizou informações técnicas do departamento de planejamento, programação, controle de produção e materiais (PPCPM) da empresa. O estudo contempla os recursos e processos destinados a atender as características da demanda dos produtos que constituem um mix de pães, responsável por $65 \%$ do faturamento da organização. Outras fontes de evidências utilizadas foram: os tempos operacionais de cada etapa; o mapeamento do fluxo do processo; o quadro de equipamentos disponíveis; as possibilidades de investimento em novos equipamentos.

Tendo por objetivo de destacar a relevância do escopo abordado nesta pesquisa foram examinadas as seguintes bases de dados, utilizando como palavra-chave: 'simulação' 'padarias': Gestão e Produção (ISSN 0104-530X); Produção (ISSN 0103-6513); Gepros (ISSN 1984-2430); Produção online (ISSN 1676-1901); Gestão Industrial (ISSN 1808-0448); Produto \& Produção; e Revista Brasileira de Pesquisa Operacional (ISSN 0101-7438). Como 
resultado da busca, ressalta-se que não foram encontrados artigos que abordam a problemática apresentada nesta pesquisa.

As próximas seções apresentam um breve referencial sobre simulação computacional e a apresentação do processo estudado. Após apresentam-se detalhes sobre a coleta e o tratamento estatístico de dados, descreve-se a metodologia do PPCPM que define o tipo e quantidade do cada produto a ser produzido pela empresa, o modelo de simulação com os indicadores utilizados e os cenários testados. O artigo finaliza com uma análise comparativa dos dados de obtidos em cada cenário e considerações sobre o uso da simulação em ambientes semelhantes e observações sobre limitações do estudo e lições aprendidas.

\section{Simulação Computacional}

Segundo Lachtermacher (2007) ao utilizar processos de modelagem para auxílio na tomada de decisão, é possível obter as seguintes vantagens: (i) os modelos forçam os decisores a tornar explícitos os objetivos; (ii) forçam a identificação, o armazenamento e a análise de relacionamento das diferentes decisões que influenciam os objetivos; (iii) forçam o reconhecimento de limitações; e (iv) permitem a comunicação de idéias para facilitar o entendimento entre grupos de trabalho.

Neste trabalho, utiliza-se a simulação computacional como ferramenta de modelagem para auxiliar na tomada de decisão. Conforme Pidd (1998) a simulação computacional é a aplicação de um modelo como base para exploração e experimentação da realidade. Prado (1999) define simulação como uma técnica que, usando o computador, procura montar um modelo que melhor represente o sistema em estudo. Assim como em outras metodologias de modelagem, a simulação é utilizada em função do seu baixo custo, maior segurança e rapidez em comparação com a realização de experimentações na realidade.

Conforme Pidd (1998) o foco de um projeto de simulação diz respeito à efetivação de um modelo para interferir questões do tipo "o que aconteceria se...?". Isto é, um modelo de simulação computacional está sujeito a entradas conhecidas e os efeitos destas entradas são observados nas saídas do modelo. Pidd (1998) relata que a maioria dos sistemas existentes poderia ser simulado, apesar de uma afirmação como tal requerer considerações sobre custo, conhecimento e poder computacional necessários para este intento. Contudo, para o êxito de um projeto de simulação, é necessário observar três aspectos (RODRIGUES, 1994): (i) Técnicos - conhecimento sobre o método de simulação, modelos estocásticos de Pesquisa Operacional, teoria de probabilidade e estatística; modelar a aleatoriedade do sistema de maneira razoável; escolher o software de simulação apropriado e utilizá-lo corretamente e usar procedimentos estatísticos apropriados para interpretar os dados de saída da simulação; (ii) Gerenciais - formular corretamente o problema e empregar boas técnicas de gerenciamento de projetos; e (iii) Precisão dos dados - obter boa informação nos procedimentos de operação do sistema e lógica de controle e estabelecer a validade e a credibilidade do modelo. Um quarto aspecto é referenciado por Vaccaro e Rodrigues (2005), relativo à capacidade de transformar resultados em informações, por meio da adequada análise estatística das saídas da simulação em elementos de suporte à decisão.

A simulação pode ser utilizada tanto para projeto e avaliação de novos sistemas como para reconfiguração física de layout ou ainda para analisar mudanças no controle ou em regras de operação de sistemas existentes. As suas aplicações têm crescido em todas as áreas, auxiliando os gestores na tomada de decisão em problemas complexos e possibilitando um melhor conhecimento dos processos nas organizações (SAKURADA e MIYAKE, 2009). Segundo Vaccaro (1999) a simulação pode ser aplicável em um grande número de situações, principalmente em sistemas em que o grau de complexidade ou até mesmo o número de alternativas de solução torna proibitiva a realização de análises exaustivas. Corroborando as afirmações referentes à aplicabilidade da simulação, apresentam-se os seguintes estudos: (i) 
Ferreira et al. (2007), aplicaram a simulação computacional em sistemas portuários, com o objetivo de testar técnicas de melhorias que influenciam no desempenho global do sistema. Uma vez construído o modelo, foi possível se avaliar a eficácia de cada técnica antes mesmo de ser implantada; (ii) Mello et al. (2006) realizaram estudos de tempos em um centro cirúrgico hospitalar, através de um modelo de simulação, com o objetivo de propor melhorias para aumentar a capacidade de atendimento dos pacientes. Trabalho similar foi realizado por Ahlert et al. (2009), em uma central de diagnósticos médicos por imagem; (iii) Hussein et al. (2009) apresentam um estudo realizado no processo produtivo de uma padaria, com o intuito de promover visando à eliminação dos desperdícios no sistema, através de um modelo de simulação, o qual permite analisar: custos, identificar os gargalos, capacidade dos equipamentos, entre outros; (iv) Ebert et al. (2009) relatam o desenvolvimento de um modelo utilizado para simular a dinâmica operacional de linhas industriais de abate de frangos, contribuindo para a previsão de variáveis como: tempo de processamento, peso vivo total, peso vivo aproveitado, peso de subproduto, peso da produção total, peso do frango inteiro, e peso total de cortes; (v) Elleuch et al. (2007) efetivaram um modelo para estudar e validar propostas de melhorias no que tange ao problema de quebra de máquina em um processo de manufatura com layout celular; (vi) Souza e Alves (2006) utilizaram a simulação para analisar o processo de produção do biodiesel, obtendo os perfis de concentração, taxa de formação e decomposição dos grupos, para a formação do biocombustível; (vii) Pontes et al. (2008) descrevem a utilização de um simulador para assessorar o gerenciamento de inventário em um centro de distribuição de peças. Segundo os mesmos autores, o simulador fornece elementos que auxilia na obtenção de resultados como: a previsão de demanda, a política de reposição das peças, o percentual de atendimento dos pedidos, os meses de inventário, entre outros; e (viii) Hachicha et al. (2007) relatam a aplicação da simulação para validar possíveis estratégias a serem utilizadas, com o intuito de fornecer um melhor resultado em ternos produtivos e financeiros, em um contexto que envolve o desenvolvimento da melhor configuração de máquinas e equipamentos para a célula de manufatura em função do mix de produtos.

A simulação computacional oferece amplos benefícios no contexto "tomada de decisão e validação de idéias". É possível observar a existência de uma vasta literatura que trata da utilização de modelagem e simulação no processo decisório, tornando-se de grande valia a sua consulta, devido à relação existente entre tomada de decisão e estratégia (ROSSONI, 2006). Entretanto, existem algumas desvantagens quando se utiliza tal metodologia. Banks (1998); Centeno e Carrillo (2001); Carson II (2004) citam as seguintes desvantagens do uso da simulação: (i) necessidade de treinamento especial para desenvolver modelos; (ii) os resultados obtidos podem ser mal interpretados ou até mesmo difíceis de serem analisados; (iii) a fase de desenvolvimento do modelo e analise, pode consumir muito tempo e apresentar um alto custo; (iv) pode ser usada inadequadamente, por exemplo, quando uma solução analítica é factível; e (v) a programação de um modelo de simulação pode se tornar altamente dispendiosa e desgastante se os recursos computacionais não forem apropriados.

Com base nos relatos apresentados, optou-se por utilizar a simulação computacional como método de auxílio no dimensionamento da capacidade do processo produtivo no contexto da pesquisa relatada neste artigo. Conforme Facchin e Sellitto (2008) um modelo pode ser uma ferramenta de apoio a decisões, pois antes que os resultados sejam efetivados na realidade, esses podem ser previstos com uma dada confiabilidade por meio da virtualidade da simulação.

No que tange à condução do estudo de simulação, a fase de desenvolvimento do modelo pode ser divida em duas partes: coletar dados e desenvolver o modelo de simulação. Essas etapas estão fortemente integradas, pois para modelar o sistema é necessário dispor de várias informações, surgindo à necessidade de coleta de dados para o início da modelagem. Durante o desenvolvimento do modelo pode ocorrer à necessidade de coleta de dados 
complementares a modelagem (CASSEL, 1996). A construção do modelo de simulação tipicamente requer ambientes apropriados para a instanciação desse tipo de artefato. No caso dos modelos de simulação de processo, softwares simuladores permitem a instanciação do modelo seguindo diferentes abordagens conceituais, tais como modelagens por processos, modelagens por atividades ou modelagens por eventos (PIDD, 1998). Ambientes para modelagens mistas, isto é, combinações das abordagens acima descritas também se encontram disponíveis. Simuladores de processos podem ainda beneficiar-se de conceitos de Modelagem Interativa Visual (Visual Interactive Modeling, VIM) e Simulação Interativa Visual (Visual Interactive Simulation, VIS), que representam, em maior ou menor grau, a possibilidade do usuário criar modelos gráficos e interagir com os mesmos durante o período de simulação (PIDD, 1998). Um exemplo de ambiente de simulação é o software Micro Saint, o qual utiliza a abordagem genérica para o desenvolvimento dos modelos que é denominada rede baseada em atividades.

\section{Metodologia}

O presente artigo pode ser caracterizado como um estudo de caso. Segundo Yin (2001) o estudo de caso possui uma vantagem distinta quando é tratada uma questão do tipo 'como' ou 'por que' acerca de um conjunto contemporâneo de acontecimentos, a respeito dos quais o pesquisador tem pouco ou nenhum controle.

No que diz respeito ao método de trabalho utilizado para efetivar a proposta desta pesquisa, destacam-se os seguintes pontos: (i) primeiramente foi realizado o mapeamento do fluxo de valor, que é definido por Rother e Shook (1999) como a resultante de ações que agregam ou não valor, necessárias para transformar matéria-prima em um produto ou serviço, passando-se por todos os processos envolvidos. Com o mapeamento, foi possível visualizar e entender o fluxo do material e de informação, enquanto cada tipo de produto segue o fluxo de valor; e (ii) para gerenciar todas as etapas envolvidas em pesquisas que fazem uso da simulação computacional, empregou-se o método proposto por Law e Kelton (2000).

A construção do modelo de simulação foi feita utilizando o software Micro Saint versão 3.2 e a análise dos resultados obtidos nos diferentes cenários testados foi realizada no software SPSS versão 20. A principal técnica utilizada para avaliar os resultados obtidos foi a Análise de Variância (ANOVA). Esse teste tem por finalidade, comparar as médias de indicadores de diferentes tratamentos (cenários de simulação) de modo a avaliar se existem, ou não, diferenças significantes. Como requisitos, deve-se respeitar a normalidade dos dados e assegurar que as matrizes de variância-covariância sejam similares entre os diferentes tratamentos. O índice de significância usado nessas análises também foi de 5\%. 


\section{Apresentação e Análise do Contexto de Estudo}

Nesta seção, apresentam-se o processo de produção de pães, os métodos utilizados para coleta e tratamento estatístico dos dados de entrada e as informações referentes à demanda dos seguintes produtos que constituem o mix responsável por $65 \%$ do faturamento da organização: Pão fatiado tipo A; Pão fatiado tipo B; Pão hot dog $30 \mathrm{~g}$; Pão hot dog $30 \mathrm{~g}-$ congelado; Pão hot dog $30 \mathrm{~g}$ in natura; Pão francês $50 \mathrm{~g}$ cliente externo; Pão francês $50 \mathrm{~g}$ cliente externo - congelado; Pão francês $50 \mathrm{~g}$ vendido no balcão; Pão francês $50 \mathrm{~g}$ vendido no balcão - congelado; Pão francês 50g in natura; Pão hot dog 50g; Pão hot dog 50g - congelado; e Pão hot dog $50 \mathrm{~g}$ in natura.

\subsection{Descrição do Processo de Produção}

É ilustrado pela Figura 1, as diferentes etapas que compõem o ambiente produtivo da empresa que subsidia a realização desta pesquisa. No intuito de distinguir o fluxo de cada tipo de pão ao longo das fases do processo de produção, as etapas dispostas no lado esquerdo da Figura 1 fazem referência à fabricação dos modelos fatiados tipo $\mathrm{A}$ e $\mathrm{B}$, sendo: (i) Amassamento; (ii) Divisão volumétria e Modelagem; (iii) Estivar e colocar nos carrinhos de transporte (iv) Crescimento (Fermentação); (v) Assamento; (vi) Resfriamento; (vii) Fatiar e embalar; e (viii) Expedição. No lado direito da Figura 1 são apresentadas as etapas referentes à produção do restante dos modelos de pães considerados pela pesquisa.

A produção inicia pela separação dos Ingredientes (matérias-primas) empregados na fabricação de cada tipo de produto. Na etapa de Amassamento ocorre a mistura das matériasprimas que foram selecionadas na etapa inicial, tendo como resultado a massa do tipo de pão que se deseja obter. Após o Amassamento, ocorre a etapa de Cilindragem que visa preparar a massa para ser processada na operação subsequente denominada Divisão volumétrica. Compete ressaltar que no processo estudado, a etapa de Cilindragem não é aplicada aos modelos fatiados.

$\mathrm{Na}$ etapa de Divisão volumétrica, a massa é dividida em porções iguais conforme a quantidade necessária para cada unidade do produto. Na Modelagem, cada pedaço de massa deve ser moldado para obter o formato característico de cada tipo de pão. Na etapa de Estivar e colocar nos carrinhos, cada tipo de massa já modelada é acondicionada em utensílios específicos e posteriormente armazenada nos carrinhos de transporte. Entretanto, precedente a etapa de Estivar e colocar nos carrinhos deve ser executada a etapa de Untar os utensílios, que tem por finalidade evitar que os pães se fixem nas superfícies dos utensílios após a etapa de Assamento, o que poderia causar problemas de remoção e marcas na superfície do pão. Vale destacar que o processo de produção dos modelos in natura (Pão hot dog 30g in natura; Pão francês $50 \mathrm{~g}$ in natura; e Pão hot dog 50g in natura) é finalizado na etapa de Estivar e colocar nos carrinhos, sendo realizada posteriormente a expedição dos produtos para o mercado consumidor. 


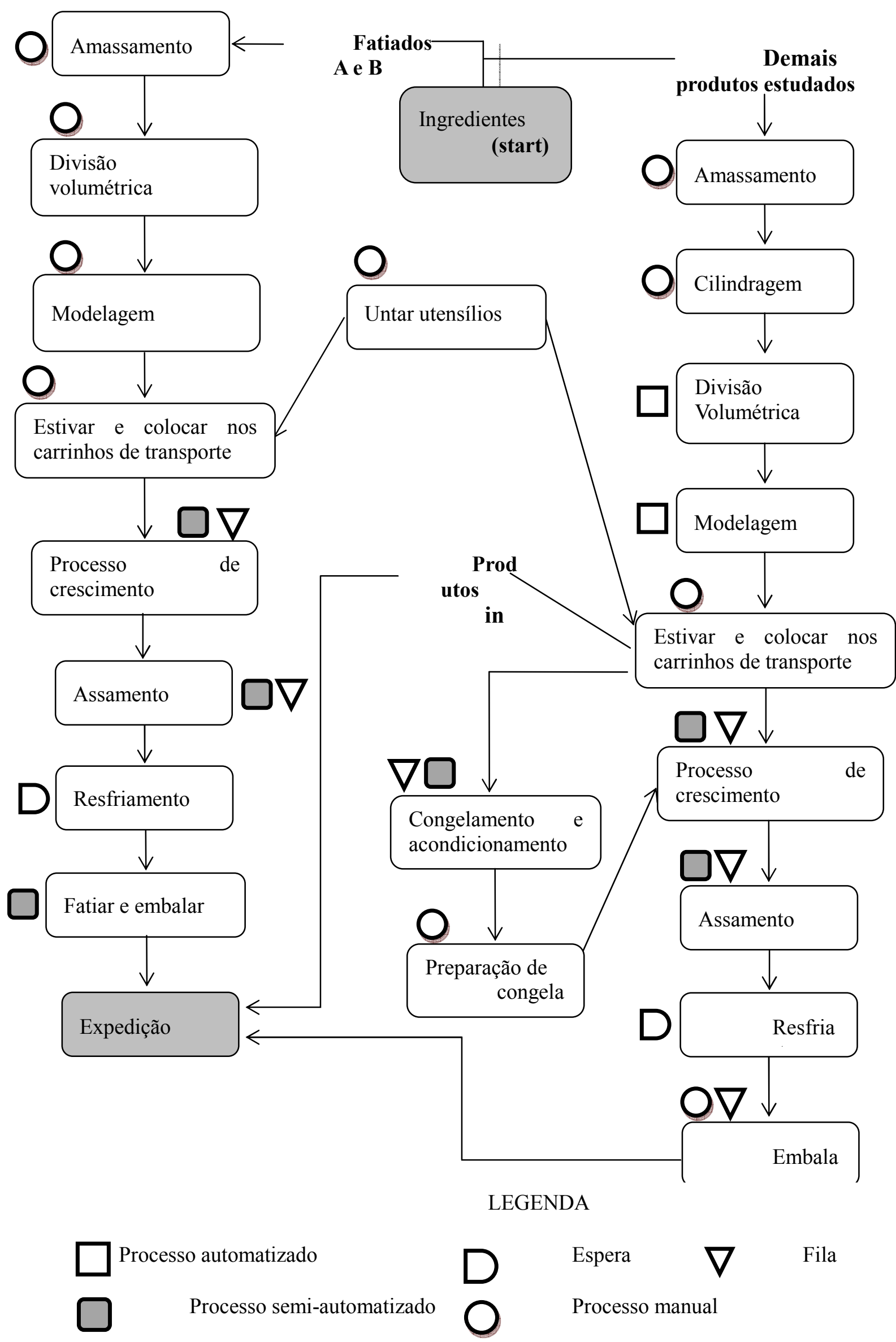

Figura 1 - Diferentes etapas que compõem o ambiente produtivo da empresa. 
Durante o processo de produção de pães, pode-se optar por congelar a massa modelada visando gerar um estoque de produtos congelados que auxilie no atendimento de eventuais solicitações de entrega não programadas ou acomodar desvios do padrão de demanda. Neste sentido, o Congelamento é realizado depois que a etapa de Estivar e colocar nos carrinhos estiver concluída. Ao término do congelamento, a massa congelada deve ser retirada dos utensílios, embalada e armazenada em câmaras frias. A etapa de congelamento é aplicada nas ordens de produção que fazem menção aos modelos: Pão hot dog $30 \mathrm{~g}$ - congelado; Pão francês $50 \mathrm{~g}$ cliente externo - congelado; Pão francês $50 \mathrm{~g}$ vendido no balcão - congelado; e Pão hot dog $50 \mathrm{~g}$ - congelado.

A etapa de Preparação de congelados é realizada apenas quando as massas que estão congeladas devem ser assadas. Para isso: (i) retira-se a massa congelada da embalagem na qual foi acondicionada; (ii) deve-se estivar a massa congelada utilizando os utensílios já untados e adequados ao modelo; e (iii) colocam-se os utensílios nos carrinhos. Em seguida, os carrinhos são colocados em um espaço apropriado, no qual a temperatura ambiente contribuirá para que a camada mais espessa do gelo evapore. Após a evaporação, os carrinhos são encaminhados para a etapa de crescimento (Fermentação).

$\mathrm{Na}$ etapa de Crescimento (Fermentação) são utilizados equipamentos específicos para realizar a fermentação da massa. Ao concluir a fermentação, é executado o Assamento, no qual a massa fermentada é levada ao forno. O Assamento estará concluído quando a massa apresentar uma coloração intensa e brilhante.

A etapa de Resfriamento consiste em um intervalo de tempo para que o pão ao sair do forno perca calor. Após o resfriamento, é realizada unicamente para os modelos fatiados a etapa de Fatiar e embalar, no entanto, para o restante dos modelos considerados neste estudo é efetivada a etapa de Embalagem, na qual os diferentes modelos recebem a embalagem característica. Por fim, ocorre a expedição dos produtos para o mercado consumidor.

\subsection{Coleta e Tratamento Estatístico dos Dados de Entrada}

Os dados de entrada formam o conjunto das informações necessárias para o modelo de simulação. Tais informações são divididas tipicamente em: (i) tempos característicos das operações; (ii) capacidades produtivas; e (iii) regras ou políticas operacionais. No contexto deste estudo, essas informações foram divididas em: (a) dados da demanda do mix de produtos; (b) capacidades produtivas dos recursos disponíveis e a serem eventualmente adquiridos; e (c) dados específicos do processo de produção de pães, tais como requisitos e esperas técnicas, além do fluxo de produção previamente apresentado.

A base de dados da demanda do mix de produtos foi construída a partir do procedimento formulado pelo departamento de planejamento, programação e controle da produção (PPCPM) da empresa. Para determinar a base de dados do processo de produção de pães, primeiramente foi verificada a existência de históricos tempos das operações. Após, foi realizado o restante da coleta das informações dos tempos do processo de fabricação, por meio de uma ficha de controle de operações, que foi desenvolvida pelos autores. Nesta ficha foram anotados os tempos de início e fim de cada uma das atividades desempenhadas pelos operadores durante o expediente de trabalho. No final do expediente, as informações coletadas foram transferidas para planilhas do software Excel e verificadas, formando uma base de dados composta pela caracterização de cada operação e pelo tempo, em minutos, ocupados para realizar as atividades necessárias para a produção dos modelos estudados. Ao término da coleta de tempos, iniciou-se a coleta das informações técnicas e constantes do processo, das quais podem ser citadas capacidades de armazenamento dos utensílios e dos carrinhos de transporte, limites de volume e peso para processamento das massas geradas nos equipamentos, número de carrinhos disponíveis para congelamento, etc. 
Ao concluir a coleta dos dados de entrada do modelo de simulação foi realizado o ajuste das distribuições de probabilidade dos dados, através da técnica de ajuste do QuiQuadrado, onde foi utilizado um nível de significância igual a 5\% (cinco por cento). Segundo Law, Averill e McComas (1994), para que os dados de saída do modelo de simulação sejam factíveis, é de suma importância utilizar distribuições de probabilidade correspondentes aos dados coletados, as quais podem ser determinadas através dos testes Qui-Quadrado ou Kolmogorov-Smirnov.

\subsection{Dados da Demanda do Mix de Produtos}

Para fins do estudo realizado foram utilizados os valores das quantidades que devem ser produzidas durante uma semana típica, conforme o departamento de PPCPM da empresa, para cada um dos produtos estudados. As informações de quanto produzir e em qual dia se deve produzir foram definidas com base nas restrições de entrega impostas pelo mercado consumidor. Conforme avalizado pela empresa, assumiu-se para fins de análise e comparação de cenários, que a capacidade de produção será estimada a partir desses valores.

No Quadro 1 são apresentadas as quantidades que devem ser produzidas durante a semana, para os modelos de Pão 'fatiado tipo A' e 'fatiado tipo B'.

\begin{tabular}{|cc|c|c|c|c|c|}
\hline \multicolumn{7}{|c|}{ Ordem de produção } \\
\hline & Produtos & Seg & Ter & Qua & Qui & Sex \\
\hline Pão fatiado tipo A & 528 & 480 & 480 & 720 & 768 \\
\hline Pão fatiado tipo B & 528 & 480 & 480 & 720 & 768 \\
\hline & Total produto pronto & $\mathbf{1 . 0 5 6}$ & $\mathbf{9 6 0}$ & $\mathbf{9 6 0}$ & $\mathbf{1 . 4 4 0}$ & $\mathbf{1 . 5 3 6}$ \\
\hline
\end{tabular}

Quadro 1 - Ordem de produção do tipo Pão fatiados (em unidades).

O Quadro 2 mostra as quantidades que devem ser produzidas em cada dia durante a semana para atender a demanda, do modelo Pão hot dog $30 \mathrm{~g}$.

\begin{tabular}{|r|c|c|c|c|c|c|}
\hline \multicolumn{7}{|c|}{ Ordem de produção } \\
\hline Produtos & Seg & Ter & Qua & Qui & Sex & Sáb \\
\hline Pão hot $\operatorname{dog} 30 \mathrm{~g}$ & 0 & 0 & 0 & 2.100 & 2.100 & 0 \\
\hline Pão hot $\operatorname{dog} 30 \mathrm{~g}-$ congelado & 2.100 & 2.100 & 2.100 & 0 & 3.150 & 3.150 \\
\hline Total produto pronto & $\mathbf{0}$ & $\mathbf{0}$ & $\mathbf{0}$ & $\mathbf{2 . 1 0 0}$ & $\mathbf{2 . 1 0 0}$ & $\mathbf{0}$ \\
\hline Total produto congelado & 2.100 & 2.100 & 2.100 & 0 & 0 & 0 \\
\hline Total produto transformado & 0 & 0 & 0 & 0 & 3.150 & 3.150 \\
\hline
\end{tabular}

O esquema de produção de Pão hot dog $30 \mathrm{~g}$ ocorre da seguinte maneira: na segunda, terça e quarta são produzidas e acondicionadas na câmara fria 2.100 unidades de Pão hot dog $30 \mathrm{~g}$ congelado em cada dia. Na quinta e sexta são produzidos e acondicionados na expedição 2.100 unidades de Pão hot dog $30 \mathrm{~g}$ (produto pronto) em cada dia. O total de congelados produzidos na segunda, terça e quarta serão transformados em produtos prontos no final da mesma semana. Na sexta, são transformados 3.150 unidades de Pão hot dog $30 \mathrm{~g}$ congelado em produto pronto e o saldo restante é transformado no sábado.

A ordem de produção do modelo Pão francês $50 \mathrm{~g}$, é apresentada no Quadro 3, sendo efetivada da seguinte maneira: o Pão francês $50 \mathrm{~g}$, destinado ao cliente externo (produto pronto) e Pão francês $50 \mathrm{~g}$ vendido no balcão (produto pronto) são produzidos e entregues no mesmo dia, nas quantidades apresentadas no Quadro 3. Entretanto, existe demanda de Pão francês $50 \mathrm{~g}$ para clientes externos e de Pão francês $50 \mathrm{~g}$ vendido no balcão também no sábado. 
No contexto produtivo, a empresa optou por não produzir pães no sábado, apenas realizando as operações para transformar massa congelada em produto pronto. Deste modo, para atender à demanda do sábado que é constituída por 600 pães vendidos no balcão e 1.200 pães que devem ser entregues no cliente externo são produzidos e acondicionados na câmara fria, 1.800 unidades de Pão francês $50 \mathrm{~g}$ congelado na segunda feira, que antecede a data de entrega.

\begin{tabular}{|l|c|c|c|c|c|c|c|}
\hline \multicolumn{7}{|c|}{ Ordem de produção } \\
\hline \multicolumn{1}{|c|}{ Produtos } & Seg & Ter & Qua & Qui & Sex & Sáb \\
\hline Pão francês 50g cliente externo - produto pronto & 1.200 & 2.400 & 2.400 & 1.800 & 1.800 & $\mathbf{1 . 2 0 0}$ \\
\hline Pão francês 50g cliente externo - congelado & $\mathbf{1 . 2 0 0}$ & 0 & 0 & 0 & 0 & 0 \\
\hline $\begin{array}{l}\text { Pão francês 50g vendido no balcão - produto } \\
\text { pronto }\end{array}$ & 1.200 & 1.200 & 1.200 & 600 & 1.200 & $\mathbf{6 0 0}$ \\
\hline Pão francês 50g vendido balcão - congelado & $\mathbf{6 0 0}$ & 0 & 0 & 0 & 0 & 0 \\
\hline Total produto pronto & $\mathbf{2 . 4 0 0}$ & $\mathbf{3 . 6 0 0}$ & $\mathbf{3 . 6 0 0}$ & $\mathbf{2 . 4 0 0}$ & $\mathbf{3 . 0 0 0}$ & $\mathbf{1 . 8 0 0}$ \\
\hline Total produto congelado & 1.800 & 0 & 0 & 0 & 0 & 0 \\
\hline Total produto transformado & 0 & 0 & 0 & 0 & 0 & 1.800 \\
\hline
\end{tabular}

A ordem de produção de uma semana, referente aos modelos in natura, é apresentada no Quadro 4.

\begin{tabular}{|c|c|c|c|c|c|}
\hline \multicolumn{6}{|c|}{ Ordem de produção } \\
\hline Produtos & Seg & Ter & Qua & Qui & Sex \\
\hline Pão francês $50 \mathrm{~g}$ in natura & 1.200 & 1.200 & 1.200 & 1.200 & 1.200 \\
\hline Pão hot dog $50 \mathrm{~g}$ in natura & 6.000 & 6.000 & 6.000 & 6.000 & 6.000 \\
\hline Pão hot dog $30 \mathrm{~g}$ in natura & 2.100 & 2.100 & 2.100 & 2.100 & 2.100 \\
\hline Total in natura & 9.300 & 9.300 & 9.300 & 9.300 & 9.300 \\
\hline
\end{tabular}

Quadro 4 - Ordem de produção in natura (em unidades).

Para o modelo de Pão hot dog $50 \mathrm{~g}$, o Quadro 5 mostra as quantidades a serem produzidas em cada dia da semana. Este modelo de pão é o que apresenta maior quantidade demandada, quando comparado aos outros modelos estudados. $\mathrm{O}$ esquema de produção ocorre de forma semelhante aos demais, não sendo descrito em detalhes. Cumpre observar apenas que os produtos prontos são comercializados no dia subsequente a sua produção.

\begin{tabular}{|r|c|c|c|c|c|c|}
\hline \multicolumn{7}{|c|}{ Ordem de produção } \\
\hline Produtos & Seg & Ter & Qua & Qui & Sex & Sáb \\
\hline Pão hot $\operatorname{dog} 50 \mathrm{~g}$ & 0 & 1.800 & 1.800 & 10.200 & 10.200 & 0 \\
\hline Pão hot $\operatorname{dog} 50 \mathrm{~g}-$ congelado & $\mathbf{9 . 6 0 0}$ & 7.800 & $\mathbf{8 . 4 0 0}$ & 0 & 13.200 & $\mathbf{1 2 . 6 0 0}$ \\
\hline Total produto pronto & $\mathbf{0}$ & $\mathbf{1 . 8 0 0}$ & $\mathbf{1 . 8 0 0}$ & $\mathbf{1 0 . 2 0 0}$ & $\mathbf{1 0 . 2 0 0}$ & $\mathbf{0}$ \\
\hline Total produto congelado & 9.600 & 7.800 & 8.400 & 0 & 0 & 0 \\
\hline Total produto transformado & $\mathbf{0}$ & $\mathbf{0}$ & $\mathbf{0}$ & & 13.200 & 12.600 \\
\hline
\end{tabular}

Quadro 5 - Ordem de produção Pão hot dog 50g (em unidades).

\section{Modelo de Simulação, Indicadores Utilizados e Cenários de Análise}

Para desenvolver o modelo de simulação foi utilizado o software Micro Saint, o qual possibilitou esquematizar em um modelo computacional, as informações coletadas e descritas na seção anterior. O modelo de simulação gerado representa uma semana típica do sistema 
produtivo, sendo o período temporal de trabalho compreendido por 24 horas/dia de trabalho (três turnos) de segunda-feira a sábado. Durante simulação, cada entidade que percorre o modelo tem a função de representar o comportamento de um lote de um determinado tipo de produto que faz parte do mix estudado. Desta forma, o usuário pode interagir com o modelo e observar as características específicas de interesse sobre o processo modelado. Contudo, apenas informações de tempos, demandas, etc., não foram suficientes para se determinar a real capacidade do processo produtivo. Regras relativas à divisão dos lotes em unidades e ao acondicionamento em carrinhos foram necessárias para representar a complexidade do processo real.

No entanto, conforme preconizado por Law e Kelton (2000) é necessário efetivar o modelo conceitual, ajustar as distribuições de probabilidade dos dados coletados e validar essas atividades preliminarmente à construção do modelo computacional. Neste enfoque, a validação do modelo conceitual ocorreu de modo qualitativo, por meio de uma apresentação das informações coletas para os especialistas do processo à luz das seguintes requisitos norteadores: (i) as informações contribuem para o objetivo do estudo; (ii) o escopo possui um período temporal delimitado para análise; (iii) apresenta a função de cada variável determinística e aleatória; e (iv) comporta a utilização dos indicadores de desempenho.

Posterior à validação do modelo conceitual foi iniciado o desenvolvimento do modelo base computacional. O modelo base foi construído ao longo de dois meses, passando por quatro versões tentativas até que as informações de saída geradas pelo modelo computacional pudessem ser consideradas válidas, no que concerne a representação do processo de modo aceitável. Este relato visa a contribuir com a literatura sobre o tema da simulação, observando que a construção de modelos de simulação requer um processo de aprendizado sobre o contexto a ser modelado. Esse processo de aprendizado demanda interação e observação crítica, para identificação de quais características da realidade podem ser desprezadas e quais são imprescindíveis para que o modelo sirva adequadamente aos propósitos do estudo realizado. Também visa a contribuir quanto à importância da compreensão dos detalhes relativos à produção de itens perecíveis, como o caso dos alimentos. Diversas regras relativas aos tempos máximos de espera dos produtos necessitaram ser explicitadas. Sua necessidade somente foi percebida pela equipe da empresa na medida em que o modelo, de forma construtiva, foi apresentado e a equipe visualizava, ainda que de forma virtual, as etapas e os potenciais desvios do processo real. Por fim, rodadas piloto com o modelo base foram realizadas a fim de gerar informações para sua validação.

No que tange a validação do modelo computacional foi realizada uma análise que consiste na comparação dos dados obtidos por observações no sistema real, com as saídas geradas pelo modelo durante a realização das rodadas piloto. Posteriormente a análise comparativa, o modelo foi verificado junto da equipe técnica da empresa, havendo um consenso entre os integrantes do projeto, quanto à capacidade de representar os elementos necessários do ambiente produtivo.

Para monitorar o comportamento do modelo simulado frente a cada cenário foi construído um sistema de indicadores de desempenho. Os indicadores utilizados para auxiliar na análise comparativa dos cenários desenvolvidos foram: (a) horário de término da produção - fornece o instante em que o último lote de um determinado tipo de produto sai do sistema de produção. Esta informação é coletada para verificar se o sistema esta operando dentro da faixa de tempo pré-estabelecida; (b) lead time - fornece o tempo gasto por um lote, para percorrer todas as operações do processo produtivo necessárias para sua fabricação, incluindo eventuais esperas. Esse indicador foi determinado de forma individualizada, para cada lote e tipo de produto; (c) utilização de recursos - percentual do tempo disponível utilizado em produção, conforme as diferentes jornadas de trabalho, para cada um dos recursos produtivos; e (d) estatísticas de fila - informações estatísticas sobre o tamanho da fila e tempo de espera dos lotes antes de cada operação. 
Com base no escopo desta pesquisa foram estruturados 3 cenários para avaliação, sendo estes, denominados A, B e C. As características de cada um dos cenários foram recomendadas pelos gestores do processo e pelos autores deste estudo, compreendendo os seguintes pontos: (i) alterações propositais nas quantidades demandadas (apresentadas nos Quadro 1 a 5) dos produtos estudados; e (ii) quantidade e capacidade de processamento dos equipamentos utilizados no processo de produção. Sob este enfoque, a proposta do cenário A utiliza os valores da demanda sem qualquer alteração e a proposta de quantidade e de capacidade dos equipamentos é descrita no Quadro 6. O cenário B propõe-se a testar um cenário futuro de aumento de $100 \%$ na demanda diária dos produtos Pão fatiado tipo A e Pão fatiado tipo B. Por fim, o cenário $\mathrm{C}$ propõe manter as características do aumento da demanda dos produtos fatiados, descrita no cenário $\mathrm{B}$ e propõe uma nova configuração de equipamentos (Quadro 6) segundo uma previsão de expansão proposta pelo corpo gestor da panificadora.

\begin{tabular}{|c|c|c|l|l|}
\hline \multicolumn{3}{|c|}{ Cenários } & \multicolumn{2}{|}{ Descrição dos equipamentos utilizados na produção } \\
\hline A & B & C & \\
\hline 1 & 1 & 1 & Quantidade de amassadeiras igual a $n$ unidades. \\
\hline 1 & 1 & 1 & Quantidade de cilindros igual a $n$ unidades. \\
\hline 1 & 1 & 1 & Quantidade de amassadeiras igual a $n$ unidades (específica para modelos fatiados). \\
\hline 3 & 3 & 3 & $\begin{array}{l}\text { Quantidade } n \text { de linhas de: divisão volumétrica, modelagem, estivar e colocar nos } \\
\text { carrinhos. }\end{array}$ \\
\hline 1 & 1 & 1 & $\begin{array}{l}\text { Quantidade } n \text { de linhas de: divisão volumétrica, modelagem, estivar e colocar nos } \\
\text { carrinhos (destinada unicamente para os modelos fatiados). }\end{array}$ \\
\hline 6 & 4 & 2 & Capacidade de processamento igual a $n$ carrinhos dentro da câmara de crescimento. \\
\hline 4 & 2 & 1 & Capacidade de processamento igual a $n$ carrinhos dentro da câmara de congelamento. \\
\hline 4 & 3 & 2 & $\begin{array}{l}\text { Capacidade de processamento igual a } n \text { carrinhos dentro da câmara de crescimento } \\
\text { (modelos fatiados). }\end{array}$ \\
\hline 4 & 3 & 2 & Quantidade $n$ de Fornos. Cada forno processa um carrinho por ciclo. \\
\hline 2 & 2 & 1 & Quantidade $n$ de Fornos. Cada forno processa um carrinho por ciclo (modelos fatiados). \\
\hline 1 & 1 & 1 & Quantidade $n$ de equipamentos de fatiar e embalar (modelos fatiados). \\
\hline
\end{tabular}

Uma vez determinadas as características de cada um dos cenários, definiu-se o número de replicações necessárias e para isso, utilizou-se o conjunto de critérios apresentados no Quadro 7. Cabe destacar que o menor número de replicações representa cerca de 14,25 meses em termos do tempo real da operação da empresa. Os modelos foram rodados em um notebook com processador T5500 de dois núcleos e 4 GB de RAM. Cada conjunto de simulações levou aproximadamente 1,4 minutos.

\begin{tabular}{|l|c|c|c|}
\hline \multicolumn{4}{|c|}{ Determinação do número de rodadas } \\
\hline \multicolumn{1}{|c|}{ Descrição } & Cenário A & Cenário B & Cenário C \\
\hline Nível de Significância & $5 \%$ & $5 \%$ & $5 \%$ \\
\hline Erro absoluto aceitável (minutos) & 3,00 & 3,00 & 4,00 \\
\hline Número de replicações iniciais & 30 & 30 & 30 \\
\hline Desvio padrão (minutos) & 11,00 & 11,00 & 16,40 \\
\hline Tamanho sugerido de replicações & 57 & 57 & 71 \\
\hline
\end{tabular}

Quadro 7 - Número de replicações feitas para cada cenário. 
Para determinar o número de rodadas da simulação foram utilizadas inicialmente 30 rodadas e a partir do desvio padrão da variável de saída 'tempo de processamento da operação de fatiar e embalar', gerado pelo modelo de simulação foi obtido o número de rodadas para cada cenário, conforme apresentado no Quadro 7. Essa variável foi selecionada para o cálculo do número de rodadas, pois apresentou o maior valor para as variáveis de tempo de processamento e que possibilitou a geração dos relatórios de saída no software Micro Saint. Contudo, compete observar que as variáveis relacionadas ao tempo de espera na fila, apresentaram valores superiores para o desvio padrão, no entanto, resultariam em um número de replicações que impossibilita a geração dos relatórios de saída por motivos de limitação de recurso computacional.

\section{Resultados Obtidos, Análise e Discussão}

Os dados de saída do modelo de simulação são fornecidos pelos indicadores de desempenho que tem a finalidade de mostrar o comportamento do modelo frente a cada um dos três cenários estudados. No entanto, para definir qual o cenário que provoca mudanças significativas no modelo, é necessário verificar a ocorrência de possíveis diferenças entre os dados de saída de cada indicador, em cada cenário. Para isso, foi utilizada a técnica de Análise Univariada de Variância (ANOVA) aplicada nos seguintes indicadores: utilização de recursos, lead time e horário de término da produção. Segundo Hair et al. (2009) procedimentos univariados de variância são utilizados para detectar diferenças entre grupos em uma única variável dependente métrica. Cabe ressaltar que ao utilizar os procedimentos de teste univariado (ANOVA) é necessário atender os seguintes pressupostos: a variável dependente deve ser normalmente distribuída e as variâncias devem ser iguais para todos os grupos de tratamento (HAIR et al., 2009). Neste estudo foi utilizado o teste de Levene para validar a homogeneidade da variância, onde a hipótese nula $\left(\mathrm{H}_{0}\right)$ do teste promove a homogeneidade (igualdade) das variâncias da população. Com base na significância obtida no teste (maior que $5 \%$ ), não foram encontradas evidências de diferença nas variâncias para cada um dos indicadores relatados anteriormente. Depois de validado os pressupostos, apresentados por Hair et al. (2009) foi aplicado o teste ANOVA, sendo apresentado a seguir os resultados e discussões.

Ao avaliar as informações referentes à: número de lotes na fila e tempo de permanência de um lote na fila, fornecidas pelo indicador 'estatística de filas', o cenário A apresenta os menores valores, quando comparado aos outros cenários. Nas operações de: congelamento, crescimento (fatiados) e assamento, a proposta do cenário A promove a não ocorrência de filas nessas operações e os tempos de permanência máximos do lote na fila até ser processado, são aceitáveis e não prejudicam as características físico-químicas e de qualidade do produto.

No cenário $\mathrm{B}$, mesmo com o aumento da demanda dos produtos fatiados, os valores médios de tamanho das filas e de tempo de permanência em média não representam grandes importâncias, contudo, o tempo máximo de espera na operação de crescimento e congelamento pode gerar o início da fermentação da massa modelada. Cabe ressaltar que não é problema congelar massa fermentada, no entanto, esta ocupará maior espaço na câmara fria. Já no cenário $\mathrm{C}$ ocorrem os maiores valores de tempos de espera do lote na fila e tamanho de fila. Se o lote ficar aguardando na 144,84 minutos em média para ser congelado a massa inicia o processo de fermentação, sendo este aspecto não desejado pelos gestores. Com o tempo médio de espera na fila igual à 325,78 minutos assar um lote de Pão fatiado, este período temporal poderá contribuir com a formação de problemas de qualidade.

Analisando de forma descritiva os valores de utilização dos equipamentos, ilustrados no Quadro 8, observa-se nos equipamentos pertencentes a linha de produção dos modelos fatiados, um aumento no percentual de utilização no cenário $\mathrm{B}$, quando confrontado com o 
cenário A. Isto por causa das diferenças entre as propostas em cada cenário, que promovem, entre outras, o aumento da demanda dos modelos fatiados e a redução de capacidade de processamento da câmara de crescimento (fatiados). No entanto, com a proposta do cenário $\mathrm{C}$, a taxa de utilização teve uma pequena queda se colacionada com o cenário B.

Para os equipamentos não destinados a produção de modelos fatiados é vista a proximidade dos valores de utilização, sendo encontrado um valor máximo de $0,06 \%$ de diferença na operação de Divisão volumétrica (automatizada), modelagem, estivar e colocar nos carrinhos, entre os cenários. Ao verificar potenciais diferenças nos valores de utilização de cada um dos equipamentos envolvidos na produção dos modelos fatiados, o teste ANOVA mostra um nível de significância menor que 5\%, indicando diferenças significativas entre os cenários A, B e C. No restante dos equipamentos estudados, o teste ANOVA indica diferenças entre os cenários apenas na atividade de Assamento.

Para os valores de tempo de atravessamentos, obtidos através do indicador "lead time" o teste ANOVA mostra diferenças significativas entre os cenários, apenas para os produtos: (a) Pão fatiado tipo A $(p=0,00)$; (b) Pão fatiado tipo B $(p=0,00)$; e (c) Pão hot dog $30 \mathrm{~g}$ congelado $(p=0,00)$. O restante dos produtos, não houve evidências para afirmar que os valores obtidos são diferentes, não importando a capacidade proposta em cada um.

\begin{tabular}{|l|c|c|c|c|}
\hline \multicolumn{4}{|c|}{ Taxa de Utilização dos Equipamentos (percentual médio) } \\
\hline \multicolumn{1}{|c|}{ Equipamentos / Cenários } & $\mathrm{A}$ & $\mathrm{B}$ & $\mathrm{C}$ & $\begin{array}{c}\text { ANOVA } \\
\text { P value }\end{array}$ \\
\hline Amassamento & 0,20 & 0,20 & 0,18 & 0,24 \\
\hline Cilindragem & 0,07 & 0,07 & 0,06 & 0,23 \\
\hline Amassamento (fatiados) & 0,12 & 0,23 & 0,21 & 0,00 \\
\hline $\begin{array}{l}\text { Divisão volumétrica, modelagem, estivar e } \\
\text { colocar nos carrinhos }\end{array}$ & 0,70 & 0,70 & 0,64 & 0,25 \\
\hline $\begin{array}{l}\text { Divisão volumétrica, modelagem, estivar e } \\
\text { colocar nos carrinhos (fatiados) }\end{array}$ & 0,13 & 0,26 & 0,24 & 0,00 \\
\hline Crescimento & 0,23 & 0,23 & 0,21 & 0,15 \\
\hline Congelamento & 0,22 & 0,22 & 0,20 & 0,51 \\
\hline Crescimento (fatiados) & 0,33 & 0,65 & 0,59 & 0,00 \\
\hline Assamento & 0,08 & 0,08 & 0,07 & 0,02 \\
\hline Assamento (fatiados) & 0,22 & 0,43 & 0,36 & 0,00 \\
\hline Fatiar e embalar (fatiados) & 0,14 & 0,28 & 0,23 & 0,00 \\
\hline
\end{tabular}

Nível de significância utilizado para corte igual a 5\%.

Quadro 8 - Taxa de utilização dos equipamentos.

A partir das informações providas do indicador Tempo utilizado, observa-se a ocorrência da seguinte questão: no cenário $\mathrm{C}$, é mantida a proposta de aumento da demanda dos Pães fatiados e reduzida à capacidade de processamento das operações de: crescimento; congelamento; crescimento (fatiados); assamento e assamento (fatiados) em relação aos outros cenários. Mas se a capacidade de processamento foi reduzida e a demanda a ser produzida tem o mesmo valor do cenário $\mathrm{B}$, a taxa de utilização deveria tender a um valor maior que as taxas dos outros cenários. Isto não ocorreu porque a houve uma mudança na sistemática de trabalho do cenário $\mathrm{C}$. Ou seja, nos cenários $\mathrm{A}$ e $\mathrm{B}$, quando é iniciado um turno de trabalho, igual à 1440 minutos, no final deste período toda a demanda que deveria ser feita naquele dia é concluída e quando se inicia um novo turno de trabalho, não há quantidade a ser produzida do dia anterior e nenhum lote na fábrica está aguardando o processamento. Já no cenário $\mathrm{C}$, ocorre a falta de capacidade para processar toda a demanda do dia em apenas 1440 
minutos e quando é iniciado um novo turno de trabalho, existem lotes espalhados pelas operações produtivas que não foram totalmente processados, devendo estes ser processados no dia posterior. Quando inicia o turno de trabalho independente do dia da semana nos cenários A e B, a linha de produção está sempre vazia, ou seja, nenhum lote está parado esperando o processamento, o que ocorre de forma diferente no cenário C. Durante os dias em que ocorre a produção de massa (de segunda à sexta) sobram lotes na produção de um dia para outro, ou seja, a linha de produção esta sempre cheia. No sábado onde não são produzidas massas de pão, todo o processo trabalha para finalizar os lotes que ficaram a ser produzidos. Em função da baixa capacidade de processamento, o tempo utilizado do cenário $\mathrm{C}$ é o maior quando comparado aos cenários $\mathrm{A}$ e $\mathrm{B}$, para todos os dias da semana. $\mathrm{O}$ teste ANOVA forneceu um valor de significância menor que 5\%, afirmando a ocorrência de diferenças potenciais no indicador de "Tempo utilizado" em cada cenário, ou seja, a capacidade proposta exerce influência neste indicador.

A partir dos resultados obtidos, ao realizar um comparativo entre os cenários, é possível destacar os seguintes tópicos, conforme delineados no Quadro 9 .

\begin{tabular}{|l|l|l|}
\hline \multicolumn{1}{|c|}{ Cenário A } & \multicolumn{1}{|c|}{ Cenário B } & \multicolumn{1}{c|}{ Cenário C } \\
\hline $\begin{array}{l}\text { Atende de forma } \\
\text { superdimensionada as } \\
\text { necessidades da demanda, } \\
\text { podendo ser também } \\
\text { aplicada à proposta de } \\
\text { aumento de 100\% na } \\
\text { demanda dos produtos } \\
\text { fatiados. }\end{array}$ & $\begin{array}{l}\text { lequipamentos para produção dos } \\
\text { modelos fatiados, com base no } \\
\text { aumento da demanda. }\end{array}$ & $\begin{array}{l}\text { Não pode ser aplicado, pois } \\
\text { os tempos médios e máximos } \\
\text { de permanência na fila } \\
\text { comprometem a qualidade do } \\
\text { produto e causam } \\
\text { modificações nas } \\
\text { características físico- } \\
\text { químicas. }\end{array}$ \\
\hline $\begin{array}{l}\text { Promove a não ocorrência } \\
\text { de filas nas operações mais } \\
\text { significativas. }\end{array}$ & $\begin{array}{l}\text { Os valores médios e máximos de } \\
\text { tempo de permanência na fila e } \\
\text { tamanho da fila nas operações de: } \\
\text { crescimento e assamento não } \\
\text { apresentam problemas e podem ser } \\
\text { aplicados. }\end{array}$ & $\begin{array}{l}\text { Não atende a necessidade de } \\
\text { finalizar todos os lotes } \\
\text { iniciados no dia, postergando } \\
\text { a data de finalização para o } \\
\text { dia subseqüente. }\end{array}$ \\
\hline $\begin{array}{l}\text { Os valores de tempo de } \\
\text { permanência médio e } \\
\text { máximo do lote na fila são } \\
\text { aceitáveis e não prejudicam } \\
\text { as características físico- } \\
\text { químicas. }\end{array}$ & $\begin{array}{l}\text { Devido ao valor de tempo de } \\
\text { permanência máxima, não se pode } \\
\text { aplicar a proposta de capacidade feita } \\
\text { pelo cenário B para a operação de } \\
\text { congelamento, pois o lote que ficar } \\
\text { aguardando o tempo máximo para ser } \\
\text { processado irá iniciar o processo de } \\
\text { crescimento da massa e o objetivo } \\
\text { não é esse. }\end{array}$ & $\begin{array}{l}\text { Devido à falta de capacidade } \\
\text { necessita de um tempo } \\
\text { utilizado maior que os outros } \\
\text { cenários para finalizar as } \\
\text { tarefas atrasadas. }\end{array}$ \\
\hline
\end{tabular}

Quadro 9 - Aplicabilidade dos cenários.

\subsection{Principais Vantagens do Uso da Simulação no Escopo da Pesquisa}

As principais vantagens obtidas neste estudo com a utilização do método de simulação foram: (i) foi possível determinar o atual quadro de máquinas de forma precisa e mensurar importantes informações como: qual é o gargalo do sistema, em quais operações ocorrem filas, tempo de permanência do lote na fila em cada operação, taxa de utilização dos recursos, tempo de atravessamento e qual é o horário que a produção finaliza o ultimo lote no sábado; (ii) a partir da definição do cenários, a empresa pode planejar precisamente o investimento necessário para construir o sistema produtivo e saber de forma confiável os resultados que vão ser obtidos; e (iii) o estudo possibilitou o planejamento de vendas, pois a empresa passou a conhecer melhor sua capacidade de atendimento as necessidades dos clientes. 
Contudo, as principais desvantagens de utilizar essa metodologia, confirmaram os relatos dos autores Banks (1998); Centeno e Carrillo (2001); Carson II (2004), pois a necessidade de tempo, pessoal capacitado para desenvolver o modelo e a dificuldade de interpretação dos dados de saída realmente ocorreram neste projeto.

\section{Conclusão}

O presente trabalho teve o objetivo de determinar a capacidade produtiva de um processo produtivo de panificação para atender às necessidades de alteração da demanda do mix de produtos. Por meio de uma abordagem de simulação computacional, foram construídos três cenários para avaliar os possíveis impactos nos indicadores, conforme a proposta de capacidade e demanda que cada cenário apresenta.

Finalmente, a aplicação de simulação no contexto estudado permitiu observar o potencial de redução dos riscos associados à tomada de decisão, principalmente em situações em que grandes volumes de recursos serão necessários. O esforço para o desenvolvimento do modelo apresentado neste estudo não foi necessariamente perdido com o fim do estudo, pois ainda permite que se criem outros cenários com o intuito de avaliar a capacidade de produção a partir de características como: aumento da demanda, entrada de novos itens no mix de produtos, etc.

Deve-se destacar ainda que seja uma aplicação, o potencial de uso de simulação é inexplorado em diversos contextos brasileiros, principalmente em pequenas e médias empresas e que estudos deste tipo contribuem academicamente na geração de casos de analise, bem como para a divulgação de métodos criteriosos de suporte à decisão, aproximando academia e ambientes aplicados.

\section{Referências}

AHLERT F. C.; de MOURA, L. C. S.; BORBA, G. S.; SILVA, D. O.; SILVA, D. D. Gestão de Serviços na Área da Saúde: a Simulação Computacional no auxílio à tomada de decisão. In: ENCONTRO NACIONAL DE ENGENHARIA DE PRODUÇÃO, 29., 2009, Salvador, BA, Brasil.

BANKS, J. Handbook of Simulation - Principies, methodology, advances, applications and pratice. New York: John Wiley \& Sons, 1998.

CARSON II, J. S. Introduction to modeling and simulation. In: WINTER SIMULATION CONFERENCE, 2004.

CASSEL R. A. (1996). Desenvolvimento de uma abordagem para divulgação da simulação no setor calçadista gaúcho. Dissertação de Mestrado apresentada ao Programa de Pós-Graduação em Engenharia de Produção (PPGEP) da Universidade Federal do Rio Grande do Sul (UFRGS).

CENTENO, M. A.; CARRILLO, M. Challenges of introducing simulation as a decision making tool. In: WINTER SIMULATION CONFERENCE, 2001.

EBERT, D. C.; SILVA, L. C.; VILAS BOAS, M. A. Simulação da dinâmica operacional de um processo industrial de abate de aves. Ciência e Tecnologia de Alimentos, Campinas, v. 29, n 2, p. 305-309, abr.-jun. 2009.

ELLEUCH, M.; MASMOUDI, F.; BEN BACHA, H.; MAALEJ, A.Y. Simulation

Manufactoring Cells with unreliable machines. Tunísia, 2007. 
FACCHIN, T.; SELLITTO, M. A. Medição do inventário em processo e tempo de atravessamento em manufatura por modelagem em redes de Petri e diagrama de resultados. Revista Gestão \& Produção, São Carlos, v. 15, n. 2, p. 307-321, maio-ago. 2008.

FERREIRA, Marcos A. M.; MENDES, Ricardo J.; CARNIERI, Celso. Análise de desempenho de sistemas portuários usando simulação matemática e estatística. Revista Produção On line, v. 7, n. 3, nov. 2007.

HACHICHA, W.; MASMOUDI, F.; HADDAR, M. An improvement of a cellular manufactoring system design using simulation analysis. Tunísia 2007.

HAIR JUNIOR, J. F.; ANDERSON, R.E.; TATHAM, R. L.; BLACK, W. C. Análise multivariada de dados. 9. ed. Porto Alegre: Bookman, 2009.

HUSSEIN, W. B.; HECKER, F.; MITZSCHERLING, M.; BECKER T. Computer Modelling and Simulation of Bakeries' Production Planning. International Journal of Food Engineering v. 5, 2009.

KLIPPEL, M.; ANTUNES JÚNIOR, J. A. V.; VACCARO, G. L. R. Matriz de posicionamento estratégico de materiais: conceito, método e estudo de caso. Gestão e Produção, v. 14, n. 1, 2007.

LAW, A. M.; KELTON, W. D. Simulation modeling and analysis. 3. ed. Boston: McGraw-Hill, 2000 .

LAW, A. M.; MCCOMAS, M. G.; VINCENT, S. G. The crucial role of input modeling in successful simulation studies - Industrial Engineering; Jul 1994; 26, 7; ABI/INFORM, Global.

LACHTERMACHER, G. Pesquisa Operacional na tomada de decisão. Rio de Janeiro: Elsevier, (2007).

MELLO, C. H. P.; TEIXEIRA, L. A. T.; SALGADO, E. G.; TORGA, B. L. M.; SCAFF, V. P. Simulação computacional: estudo de tempos em um centro cirúrgico. In: ENEGEP, 26., 2006, Fortaleza, CE, Brasil. Anais eletrônicos.

PIDD, M. Modelagem empresarial: ferramentas para tomada de decisão. 1. ed. Porto Alegre: Bookman, 1998.

PONTES, H. L. J.; PALMA, J. G.; PORTO, A. J. V. SIMULINVE - um simulador de inventário para um centro de distribuição de peças. Produção On line. v. 8, n. 3, 2008.

PRADO, D. S. Teoria das filas e da Simulação. 2. ed. Belo Horizonte: Desenvolvimento Gerencial, 1999.

SAKURADA, N.; MIYAKE, D. I. Aplicação de simuladores de eventos discretos no processo de modelagem de sistemas de operações de serviços. Revista Gestão \& Produção, São Carlos - SP, v. 16, n. 1, p. 25-43, jan.-mar. 2009.

SOUZA, J. S.; ALVES, J. J.N. Modelagem e Simulação da Produção do Biodiesel em Processo de Batelada. I Congresso da Rede Brasileira de Tecnologia do Biodiesel, 2006.

RODRIGUES, L. H. Developing an approach to help companies synchronize manufacturing. Lancaster University. Lancaster: s.n., 1994. Tese de Doutorado.

ROSSONI, L. Modelagem e simulação soft em estratégia. Produção on line. Vol. 6/ Num. $2 /$ Agosto de 2006.

ROTHER, M.; SHOOK, J. Aprendendo a Enxergar - Mapeando o fluxo de valor para agregar valor e eliminar o desperdício. São Paulo: Lean Institute Brasil, 1999. 
VACCARO, G. L. R.. Modelagem e Análise de Dados em Simulação. Exame de qualificação PPGC - UFRGS. Universidade Federal do Rio Grande do Sul. 1999.

VACCARO, G. L. R.; RODRIGUES, L. H. Características Desejadas em Softwares de Análise de Resultados de Simulação. In: ENEGEP, 25., 2005, Porto Alegre, RS, Brasil.

YIN, R. K. Estudo de caso: planejamento e métodos. Porto Alegre: Bookman, 2001. 